\title{
Correction: Nationwide survey for current clinical status of amniocentesis and maternal serum marker test in Japan
}

\author{
Hidehiko Miyake ${ }^{1}$ Shigehito Yamada ${ }^{1}$ Yosuke Fujii ${ }^{2} \cdot$ Hideaki Sawai $^{3} \cdot$ Naoko Arimori $^{4} \cdot$ Yasuko Yamanouchi $^{5}$. \\ Yuka Ozasa $^{6} \cdot$ Makoto Kanai $^{7} \cdot$ Haruhiko Sago $^{8} \cdot$ Akihiko Sekizawa $^{9} \cdot$ Fumio Takada $^{10}$ - Hideaki Masuzaki ${ }^{11}$. \\ Yoichi Matsubara ${ }^{12} \cdot$ Fumiki Hirahara $^{13} \cdot$ Koji Kugu $^{14}$
}

Received: 3 August 2018 / Accepted: 3 August 2018 / Published online: 23 August 2018

(c) The Author(s) under exclusive licence to The Japan Society of Human Genetics 2018

Correction to: Journal of Human Genetics. 2016 Oct;61 (10):879-884 https://doi.org/10.1038/jhg.2016.67; published online 30 June 2016
Since the publication of this paper, the authors noticed that Yosuke Fujii was assigned to an incorrect affiliation. The affiliation information is provided correctly below.

The authors would like to apologize for this mistake.
Hidehiko Miyake

miyake.hidehiko@ocha.ac.jp

1 Clinical Genetics Unit, Kyoto University Hospital, Kyoto, Japan

2 Kyoto University Hospital, Kyoto, Japan

3 Department of Obstetrics and Gynecology, Hyogo College of Medicine, Nishinomiya, Japan

4 The School of Health Sciences, Niigata University, Niigata, Japan

5 Department of Social Work, Faculty of Health and Welfare, Kawasaki University of Medical Welfare, Kurashiki, Japan

6 Department of Nursing, Tokyo Medical and Dental University Medical Hospital, Tokyo, Japan

7 Department of Family and Child Nursing, and Midwifery, Shinshu University School of Health Sciences, Matsumoto, Japan

8 Center of Maternal-Fetal, Neonatal and Reproductive Medicine,
National Center for Child Health and Development, Tokyo, Japan

9 Department of Obstetrics and Gynecology, School of Medicine, Showa University, Tokyo, Japan

10 Department of Medical Genetics and Genomics, Kitasato University Graduate School of Medical Sciences, Sagamihara, Japan

11 Department of Obstetrics and Gynecology, Nagasaki University, Nagasaki, Japan

12 National Research Institute for Child Health and Development, Tokyo, Japan

13 National Hospital Organization Yokohama Medical Center, Yokohama, Japan

14 Department of Obstetrics and Gynecology, Tokyo Metropolitan Bokutoh Hospital, Tokyo, Japan 